\title{
Optimal control of nonlocal fractional evolution equations in the $\alpha$-norm of order
} $(1,2)$

\author{
Azmat Ullah Khan Niazi ${ }^{1,2}$, Naveed lqbal ${ }^{3 *}$ and Wael W. Mohammed ${ }^{3,4}$
}

\section{"Correspondence:}

naveediqbal1989@yahoo.com; n.iqbal@uoh.edu.sa

${ }^{3}$ Department of Mathematics,

Faculty of Science, University of Ha'il, Ha'ill, 81481, Saudi Arabia

Full list of author information is available at the end of the article

\begin{abstract}
This paper investigates the optimal control for a class of nonlocal fractional evolution equations of order $\gamma \in(1,2)$ in Banach spaces. An adequate definition of $\alpha$-mild solutions is obtained and the existence, uniqueness and continuous dependence of $\alpha$-mild solutions for the presented control system are also established. The existence of optimal pairs of nonlocal fractional evolution systems is also demonstrated with a view on the construction of the Lagrange problem. Finally, an example is propounded for the presentation of optimal control.
\end{abstract}

MSC: $26 \mathrm{~A} 33 ; 34 \mathrm{~K} 37$

Keywords: Fractional evolution equations; $\alpha$-Mild solutions; Existence; Continuous dependence; Optimal control

\section{Introduction}

Applied linguistic mathematics has the sub-branch of the theory of fractional differential equations. Ordinary and differential equations involving fractional derivatives have undergone substantial development in the present era as is evident from the monographs of Podlubny [1], Kilbas et al. [2], Zhou [3, 4] and papers [5-9] and the references therein.

The development of mild solutions is becoming the imperative cynosure of the present era for fractional differential and integro-differential equations of the order $\gamma \in(1,2)$. $\mathrm{Li}$ et al. [10] vehemently claim that fractional evolution issues with Riemann-Liouville derivative be handled by implementing the concept of resolvent family. Based on the concept of the sectorial operator, Shu [11] minutely studied the existence and uniqueness of mild solutions for nonlocal fractional differential equations. Through the implementation of analytic solution operators, $\mathrm{Li}$ [12] examined the regularity of moderate solutions of fractional abstract Cauchy problems. In addition, Kian and Yamamoto [13] analyzed the nature and estimation of solutions for semilinear fractional wave equations through Strichartz's value expansion process in bounded domains. Li et al. [14] put forward his opinion on the existence and uniqueness of fractional abstract Cauchy problems with order $\gamma \in(1,2)$. Besides, some other engaging and imperative controllability results on the

(c) The Author(s) 2021. This article is licensed under a Creative Commons Attribution 4.0 International License, which permits use, sharing, adaptation, distribution and reproduction in any medium or format, as long as you give appropriate credit to the original author(s) and the source, provide a link to the Creative Commons licence, and indicate if changes were made. The images or other third party material in this article are included in the article's Creative Commons licence, unless indicated otherwise in a credit line to the material. If material is not included in the article's Creative Commons licence and your intended use is not permitted by statutory regulation or exceeds the permitted use, you will need to obtain permission directly from the copyright holder. To view a copy of this licence, visit http://creativecommons.org/licenses/by/4.0/. 
fractional differential systems were addressed in [15] with order $\gamma \in(0,1)$ and in $[16,17]$ with order $\gamma \in(1,2)$.

The optimal control of fractional evolution equations is of paramount importance and it plays a pivotal role in various fields of science like engineering and economy and some other branches: physics, biology, electronic media, and dynamic processes. By dint of the pressing need of optimal control of fractional evolution equation in the sub-fields of the theory and its applications become better understood. In 2010, Wang et al. [18] worked on a class of fractional evolution equations, they studied the existence of a mild solution for semilinear fractional evolution equations and the optimal control equipped with the $\alpha$-norm. Wang et al. [19] extended their interesting results and worked on a group of fractional delay nonlinear integro-differential controlled systems coupled with an analytic semigroup in Banach spaces. All this work was performed on the fractional derivative of the order of $(0,1)$. For further study we refer to [20-23]. After these researches, some scholars contributed work to extend the order with $(1,2)$. For example, the authors studied the controlled time-fractional equation of Sobolev type of Caputo and Riemann-Liouville fractional derivatives with the order of $(1,2)$, respectively [24]. The authors of [25] considered approximate controllability for a class of fractional stochastic wave equations and, in [26], the existence of a mild solution and the compactness of a set of mild solutions to a nonlocal problem of fractional evolution inclusions are established. Very recent studies with new results on the controllability of fractional evolution systems with order $\gamma \in(1,2)$ have been presented in [27-31]. This paper investigates some motivating results of mild solutions to fractional evolution systems in Banach spaces.

In this research paper, the research that used alpha norms of cosine families on operators and found new results one makes good use of these outcomes for the solution of the present research paper. Further details of alpha norms of cosine families can be found in [32].

Consider the nonlinear fractional evolution system with nonlocal condition as follows:

$$
\left\{\begin{array}{l}
{ }_{0}^{c} D_{t}^{\gamma} E w(t)+G w(t)=a(t) J(t, w(t))+K(t) v(t), \quad t \in \Omega=[0, S], \\
w(0)=w_{0}+g(w), \quad w^{\prime}(0)=w_{1},
\end{array}\right.
$$

where ${ }_{0}^{c} D_{t}^{\gamma}$ is the Caputo fractional derivative of order $\gamma \in(1,2)$, the operator $-G E^{-1}$ : $D\left(G E^{-1}\right) \rightarrow U$ is the infinitesimal generator of strongly continuous cosine family $\{\mathcal{C}(t)\}_{t \geq 0}$ on a separable reflexive Banach space $U$. The function $J$ has a $U^{\alpha}$-value, $w(t):[0, S] \rightarrow U^{\alpha}$ where $U^{\alpha}=D\left(G_{b}^{\alpha} E^{-1}\right)$ is a Banach space having the norm $\|w\|_{\alpha}=\left\|G_{b}^{\alpha} w\right\|$ for $w \in U, v$ is taking values from a separable reflexive Banach space $V, K$ is a linear operator and maps $V$ into $U, a \in L^{p_{1}}\left([0, S], R^{+}\right), p_{1}>1, g: C([0, S] ; U) \rightarrow U, w_{0}, w_{1}$ are elements of the space $U$. $G$ and $E$ are linear operators. $G$ is closed, $D(E) \subset D(G)$. $E$ is bijective. The linear operator $E^{-1}: V \rightarrow D(E) \subset U$ is compact (which implies that $E^{-1}$ is bounded). It implies that $E$ is closed: since $E^{-1}$ is closed and injective, its inverse is also closed. We obtain the boundedness of linear operators $G E^{-1}: Y \rightarrow Y$. Consequently $-G E^{-1}$ generates a cosine family $\{\mathcal{C}(t), t \geq 0\}$. In considering the case of $\alpha \in(0,1)$, we know from the references that there is a similar representation of mild solutions if we have the initial value $x_{1}=$ 0 for the case of $\alpha \in(1,2)$. However, the biggest difference is that the operator $-G E^{-1}$ (typically the Laplacian operator) generates a $C_{0}$-semigroup, and one can use the method of semigroup theory to obtain some well-known results for the case of $\alpha \in(0,1)$, instead 
of cosine families. Furthermore, if $\alpha$ tends to 1 , the method of semigroup theory can be also used to deal with first-order evolution problems; if $\alpha$ tends to 2, we can directly solve an evolution problem by using the concept of cosine families. Thus, the studied evolution problem in Eq. (1) is more different from the case of $\alpha \in(0,1]$, and it is valuable to consider the existence of Eq. (1).

As far as the real-life application of the studied system is concerned, the first of concern is food technology. The problem is to determine the time-dependent optimal temperature inside a sterilizing chamber for canned foods. The industrial sterilization of canned foods is a process in which there usually occurs a degradation of nutrients and a deterioration of qualitative properties due to the temperature to which the food is overexposed in order to ensure destroying pathogenic microorganisms. We face this problem using optimal control methods. Linear constraints on the control and nonlinear ones on the state will appear as a consequence of the technological restrictions and the requirements of quality and economy. Besides an existence result for the optimal control and an optimality system, we give numerical results for some real industrial examples. They show that present industrial sterilization processes can be improved in terms of saving energy and nutrient retention. The second problem is from environmental engineering. We deal with the design and management of wastewater treatment systems discharging polluting effluent into an aquatic medium. This problem is formulated as a point-wise optimal control problem with state and control constraints. The main difficulties arise from the lack of regularity of the source term in the state system and the point-wise constraints on the state variables. We develop a theoretical analysis of the problem, propose an algorithm for its numerical resolution and give results for a realistic problem. The third problem is related to acoustic engineering, more precisely, to noise reduction by active control methods. During the last two decades, there has been an higher-growing level of interest in the control of sound by active techniques. Many of the physical principles involved have long ago been established, but the technological means for the successful implementation of active noise control, namely the fast-digital signal processors (DSP), have only recently become available. Noise reduction is based on the destructive interference of waves. Accordingly, the control consists of a (secondary) source of noise the properties of which have to be determined optimally to cancel the original undesired (primary) noise. Many optimization problems in science and engineering can be described by optimal control problems such as the control of a spacecraft or aircraft, of a chemical reaction, or of an industrial robot. Thereby, the consideration of different constraints is very important when realistic models are to be solved.

In this paper, we investigate the optimal control for the fractional evolution system (1) with nonlocal conditions. The research has been managed as follows below. Section 2 refers to a variety of notations and beneficial notions for cosine family and fractional calculus. In Sect. 3, this study is based on a changed hypothesis and, furthermore, it works on a mild solution for the system (1) which is correlated with the probability density function and cosine families. In operator families, this paper will introduce an operator that is different from some previously mentioned papers. Before going on to the next step, it is mentionable that this operator is linear bounded. Furthermore, the study proves the system is mildly solvable and mild solution is unique along with the continuous dependence of data in Sect. 4. We will prove the existence of fractional optimal controls for the 
Lagrange problem in Sect. 5. Finally, an example is propounded for the presentation of optimal control.

\section{Preliminaries}

Let $U$ and $V$ be two separable reflexive Banach spaces, equipped with the norms $|\cdot|$ and $|\cdot|_{v}$, respectively. $\mathfrak{L}(U, V)$ illustrates the space of all bounded linear operators from $U$ to $V$ equipped with the norm $\|\cdot\|_{\mathfrak{L}(U, V)}$. In particular, when $U=V$, we set $\mathfrak{L}(U, V)=\mathfrak{L}(U)$ and $\|\cdot\|_{\mathfrak{L}(U, V)}=\|\cdot\|_{\mathfrak{L}(U)}$. Typically, we put forward the fractional power operator $G_{b}^{\alpha}\left(0<\alpha<\frac{1}{2}\right)$ (see [32]) having a dense domain $D\left(G_{b}^{\alpha}\right)$ which is provided with graph norm $\|\cdot\|_{\alpha}$ to be a fractional power space $U^{\alpha}$. Then $U_{\beta} \hookrightarrow U_{\alpha}$ for $0<\alpha<\beta<\frac{1}{2}$. It is easy to see that $U^{\alpha}$ is separable from the separability of $U$. Moreover, $G_{b}^{\alpha}$ has the following basic properties.

For $S>0$, denote $\Omega=[0, S]$. Let $C\left(\Omega, U^{\alpha}\right)$ be the Banach space of continuous functions from $\Omega$ to $U^{\alpha}$ with the usual sup-norm. For brevity we denote $C\left(\Omega, U^{\alpha}\right)$ simply by $C_{0, S, \alpha}$ and its norm is given by $\|\cdot\|_{0, S, \alpha}$. We shall also make use of $\|J\|_{L^{p}\left(\Omega, R^{+}\right)}$to denote the $L^{p}\left(\Omega, R^{+}\right)$norm of $J$ whenever $J \in L^{p}\left(\Omega, R^{+}\right)$for some $p$ with $1<p<\infty$. We denote the resolvent set of $G$ by $\rho(G)$ and the resolvent of $G$ by $R(\mu, G)=\left(\mu I+G E^{-1}\right)^{-1} \in \mathfrak{L}(U)$.

Recalling the definitions and properties of fractional calculus, we refer to $[1,2]$.

The fractional integral of order $\gamma \in \mathbb{R}_{+}$with the lower limit zero for a function $u$ is defined as

$$
I_{0+}^{\gamma} w(t)=\left(g_{\gamma} * w\right)(t)=\frac{1}{\Gamma(\gamma)} \int_{0}^{t}(t-s)^{\gamma-1} w(s) d s,
$$

provided the right-hand side is point-wise defined on $[0, \infty)$, where $\Gamma$ is the Euler gamma function, the symbol $*$ denotes convolution,

$$
g_{\gamma}(t)=\frac{t^{\gamma-1}}{\Gamma(\gamma)}, \quad \text { if } t>0 ; \quad g_{\gamma}(t)=0, \quad \text { if } t \leq 0
$$

At the origin, the Dirac measure is denoted by $g_{0}(t)=\delta(t)$, when $\gamma=0$.

The Riemann-Liouville fractional derivative of order $\gamma \in \mathbb{R}_{+}$for a function $w:[0, \infty) \rightarrow$ $\mathbb{R}$ is defined by

$$
{ }_{0}^{L} D_{t}^{\gamma} w(t)=\frac{d^{n}}{d t^{n}}\left(g_{n-\gamma} * w\right)(t), \quad t \geq 0, n-1<\gamma<n,
$$

and the Caputo derivative of order $\gamma \in \mathbb{R}_{+}$for a function $w:[0, \infty) \rightarrow \mathbb{R}$ is defined by

$$
{ }_{0}^{c} D_{t}^{\gamma} w(t)={ }_{0}^{L} D_{t}^{\gamma}\left(w(t)-\sum_{k=0}^{n-1} \frac{w^{(k)}(0)}{k !} t^{k}\right), \quad t \geq 0, n-1<\gamma<n .
$$

Lemma 2.1 (See [19, Lemma 2.8]) A measurable function $X: \Omega \rightarrow U$ is a Bochner integral if $\|X\|$ is Lebesgue integrable.

Lemma 2.2 (See [33, Lemma 1.2]) Suppose that for $w \in C_{0, S, \alpha}$ we have the following inequalities:

$$
\|w(t)\|_{\alpha} \leq c+d \int_{0}^{t}(t-y)^{z-1}\|w(y)\|_{\alpha} d y, \quad t \in \Omega,
$$


with constant $c, d \geq 0$. Then there exists a constant $M^{*}>0$ independent of $c$ such that

$$
\|w(t)\|_{\alpha} \leq M^{*}(c), \quad \text { for all } t \in \Omega \text {. }
$$

Lemma 2.3 (See [34, Problem 23.9]) For each $\varphi \in L^{p}(\Omega, U)$ with $1 \leq p<+\infty$, we have

$$
\lim _{h \rightarrow 0} \int_{0}^{S}\|\varphi(t+h)-\varphi(t)\|^{p} d t=0
$$

where $\varphi(y)=0$ for $y$ not belonging to $\Omega$.

We briefly examine the concept and few properties of the cosine family. One can find more information in [35-37]. We use the following definition of the cosine family.

Definition 2.1 A one-parameter family $\{\mathcal{C}(t)\}_{t \geq 0}$ of bounded linear operators mapping the Banach space $U$ into itself is called a strongly continuous cosine family if and only if $\mathcal{C}(0)=I$ (the identity operator), $\mathcal{C}(s+t)+\mathcal{C}(s-t)=2 \mathcal{C}(s) \mathcal{C}(t)$ for all $s, t \geq 0$ and $\mathcal{C}(t) x$ is continuous in $t$ on $[0, \infty)$ for each fixed point $x \in U$.

The strongly continuous sine family $\{\mathcal{S}(t)\}_{t \geq 0}$ associated with the cosine family is defined by

$$
\mathcal{S}(t) x=\int_{0}^{t} \mathcal{C}(s) x d s, \quad x \in U, t \geq 0
$$

Assumption 2.1 ([32, Assumption 1, p. 2]) Let $b>\omega$. Then $\mathcal{S}(t) U \in D\left(G_{b}^{\frac{1}{2}}\right)$, and $G_{b}^{\frac{1}{2}} \mathcal{S}(t)$ is a strongly continuous function of the argument $t$ on $-\infty<t<+\infty$

Theorem 2.4 ([32, Lemma (1-3), Theorem 1]) For any $\alpha \in\left[0, \frac{1}{2}\right]$ there exists $C_{\alpha}>0$ such that, for every $t \geq 0,\|\mathcal{S}(t)\|_{\alpha}=\left\|G_{b}^{\alpha} \mathcal{S}(t)\right\| \leq C_{\alpha}(1+t) e^{\omega t}$.

\section{Existence and uniqueness}

In this section, the existence and uniqueness of the system (1) will be proved. For this purpose, we suppose that $G E^{-1}$ is the infinitesimal generator of a strongly continuous cosine family of uniformly bounded linear operators $\{\mathcal{C}(t)\}_{t \geq 0}$ in a separable reflexive Banach space $U$, that is, there exists $M \geq 1$ such that $\|\mathcal{C}(t)\|_{\mathfrak{L}(U)} \leq M$, for $t \geq 0$. For convenience, we also set $z=\gamma / 2$ with $\gamma \in(1,2)$.

We make use of the following assumptions:

(H1) The function $J: \Omega \times U^{\alpha} \rightarrow U$ satisfies:

(i) for each $w \in U^{\alpha}, t \rightarrow J(t, w(t))$ is measurable;

(ii) for arbitrary $\zeta_{1}, \zeta_{2} \in U^{\alpha}$ satisfying $\left\|\zeta_{1}\right\|_{\alpha},\left\|\zeta_{2}\right\|_{\alpha} \leq \sigma$, there exists a constant $L_{J}(\sigma)>0$ such that

$$
\left\|J\left(t, \zeta_{1}\right)-J\left(t, \zeta_{2}\right)\right\| \leq L_{J}(\sigma)\left(\left\|\zeta_{1}-\zeta_{2}\right\|_{\alpha}\right), \quad \text { for all } t \in \Omega
$$

(iii) there exists a constant $C_{J}>0$ such that

$$
\| J\left(t, \zeta \| \leq C_{J}\left(1+\|\zeta\|_{\alpha}\right), \quad \text { for all } \zeta \in U^{\alpha} t \in \Omega\right. \text {. }
$$


(H2) $g: C\left(\Omega, U^{\alpha}\right) \rightarrow U$ is

(i) for arbitrary $\zeta_{1}, \zeta_{2} \in U^{\alpha}$ satisfying $\left\|\zeta_{1}\right\|_{\alpha},\left\|\zeta_{2}\right\|_{\alpha} \leq \sigma$, there exists a constant $L_{g}(\sigma)>0$ such that

$$
\left\|g\left(t, \zeta_{1}\right)-g\left(t, \zeta_{2}\right)\right\| \leq L_{g}(\sigma)\left\|\zeta_{1}-\zeta_{2}\right\|_{C} \quad \text { for all } t \in \Omega
$$

(ii) continuous and compact function, and there exist constant $N_{g 1}, N_{g 2}$ such that

$$
\|g(\zeta)\| \leq N_{g 1}\|\zeta\|_{C}+N_{g 2} \quad \text { for } \zeta \in C\left(\Omega, U^{\alpha}\right)
$$

(H3) $K \in L^{b}\left(L^{p}(\Omega, V), L^{p}(\Omega, U)\right), 1<p<\infty$.

(H4) $P_{a d}=L^{p}(\Omega, V)$.

By virtue of the method of [2], one can find that Eq. (1) has the following representation:

$$
\begin{aligned}
E w(t)= & E\left(w_{0}+g(w)\right)+E w_{1} t \\
& +\frac{1}{\Gamma(\gamma)} \int_{0}^{t}(t-y)^{\gamma-1}[-G w(y)+a(y) J(y, w(y))+K(y) v(y)] d y, \quad t \in \Omega
\end{aligned}
$$

provided the right-hand side of the above equation holds.

In the sequel, we will use the probability density function $\vartheta_{z}(\theta)$ defined on $] 0, \infty$ [ as

$$
\begin{aligned}
& \vartheta_{z}(\theta)=\frac{1}{z \theta^{(1+1 / z)}} \varpi_{z}\left(\theta^{-1 / z}\right) \geq 0, \quad z \in(0,1), \\
& \varpi_{z}(\theta)=\frac{1}{\pi} \sum_{n=1}^{\infty}(-1)^{n-1}(\theta)^{-z n-1} \frac{\Gamma(n z+1)}{n !} \sin (n \pi z) .
\end{aligned}
$$

Lemma 3.1 If Eq. (2) holds, then for $t \in \Omega, z=\gamma / 2$

$$
\begin{aligned}
w(t)= & \mathcal{S}_{E}(t) E\left(w_{0}+g(w)\right)+\chi_{E}(t) E w_{1}+\int_{0}^{t}(t-y)^{z-1} \mathcal{P}_{E}(t-y) a(y) J(y, w(y)) d y \\
& +\int_{0}^{t}(t-y)^{z-1} \mathcal{P}_{E}(t-y) K(y) v(y) d y, \quad t \in \Omega,
\end{aligned}
$$

where

$$
\begin{aligned}
& \mathcal{S}_{E}(t)=\int_{0}^{\infty} E^{-1} \vartheta_{z}(\theta) \mathcal{C}\left(t^{z} \theta\right) d \theta, \\
& \chi_{E}(t)=\int_{0}^{t} \mathcal{S}_{E}(y) d y, \\
& \mathcal{P}_{E}(t)=z \int_{0}^{\infty} E^{-1} \theta \vartheta_{z}(\theta) \mathcal{S}\left(t^{z} \theta\right) d \theta .
\end{aligned}
$$

Proof For $\mu>0$. Applying the Laplace transform to (2), we get

$$
E l(\mu)=\frac{1}{\mu} E\left(w_{0}+g(w)\right)+\frac{1}{\mu^{2}} E w_{1}-\frac{1}{\mu^{\gamma}} G l(\mu)+\frac{1}{\mu^{\gamma}} m(\mu)+\frac{1}{\mu^{\gamma}} n(\mu),
$$


where

$$
\begin{aligned}
& l(\mu)=\int_{0}^{\infty} e^{-\mu y} w(y) d y, \quad m(\mu)=\int_{0}^{\infty} e^{-\mu y} a(y) J(y, w(y)) d y, \quad \text { and } \\
& n(\mu)=\int_{0}^{\infty} e^{-\mu y} K(y) v(y) d y,
\end{aligned}
$$

this implies

$$
E\left(\mu^{\gamma} I+G E^{-1}\right) l(\mu)=\mu^{\gamma-1} E\left(w_{0}+g(w)\right)+\mu^{\gamma-2} E w_{1}+m(\mu)+n(\mu) .
$$

Therefore, by the relationship between resolvent and cosine function, i.e., for $\operatorname{Re} \mu>0$,

$$
\mu R\left(\mu^{2} ; G\right) x=\int_{0}^{\infty} E^{-1} e^{-\mu t} \mathcal{C}(t) x d t, \quad R\left(\mu^{2} ; A\right) x=\int_{0}^{\infty} E^{-1} e^{-\mu t} \mathcal{S}(t) x d t, \quad x \in U
$$

we first have

$$
\begin{aligned}
E l(\mu)= & \mu^{\gamma-1}\left(\mu^{\gamma} I+G E^{-1}\right)^{-1} E\left(w_{0}+g(w)\right)+\mu^{\gamma-2}\left(\mu^{\gamma} I+G E^{-1}\right)^{-1} E w_{1} \\
& +\left(\mu^{\gamma} I+G E^{-1}\right)^{-1} m(\mu)+\left(\mu^{\gamma} I+G E^{-1}\right)^{-1} n(\mu) \\
= & \mu^{\frac{\gamma}{2}-1} \int_{0}^{\infty} E^{-1} e^{-\mu^{\frac{\gamma}{2}} y} \mathcal{C}(y) E\left(w_{0}+g(w)\right) d y+\mu^{-1} \mu^{\frac{\gamma}{2}-1} \int_{0}^{\infty} E^{-1} e^{-\mu^{\frac{\gamma}{2}} y} \mathcal{C}(y) E w_{1} d y \\
& +\int_{0}^{\infty} E^{-1} e^{-\mu^{\frac{\gamma}{2}} y} \mathcal{S}(y) m(\mu) d y+\int_{0}^{\infty} E^{-1} e^{-\mu^{\frac{\gamma}{2}} y} \mathcal{S}(y) n(\mu) d y .
\end{aligned}
$$

As $z=\gamma / 2 \in(1 / 2,1)$, so we take

$$
\begin{aligned}
l(\mu)= & \mu^{z-1} \int_{0}^{\infty} E^{-1} e^{-\mu^{z} y} \mathcal{C}(y) E\left(w_{0}+g(w)\right) d y+\mu^{-1} \mu^{z-1} \int_{0}^{\infty} E^{-1} e^{-\mu^{z} y} \mathcal{C}(y) E w_{1} d y \\
& +\int_{0}^{\infty} E^{-1} e^{-\mu^{z} y} \mathcal{S}(y) m(\mu) d y+\int_{0}^{\infty} E^{-1} e^{-\mu^{z} y} \mathcal{S}(y) n(\mu) d y
\end{aligned}
$$

Now consider the one-sided probability density function given in (3) whose Laplace transform is

$$
\left.\int_{0}^{\infty} e^{-\mu \theta} \varpi_{z}(\theta) d \theta=e^{-\mu^{z}}, \quad z \in\right] 0,1[
$$

from (5) and using (6), we have

$$
\begin{aligned}
\mu^{z-1} & \int_{0}^{\infty} E^{-1} e^{-\mu^{z} y} \mathcal{C}(y) E\left(w_{0}+g(w)\right) d y \\
& =\int_{0}^{\infty} \mu^{z-1} E^{-1} e^{-(\mu t)^{z}} \mathcal{C}\left(t^{z}\right) z t^{z-1} E\left(w_{0}+g(w)\right) d t \\
& =\int_{0}^{\infty} z(\mu t)^{z-1} E^{-1} e^{-(\mu t)^{z}} \mathcal{C}\left(t^{z}\right) E\left(w_{0}+g(w)\right) d t \\
& =\int_{0}^{\infty} \frac{-1}{\mu} \frac{d}{d t} E^{-1}\left(e^{-(\mu t)^{z}}\right) \mathcal{C}\left(t^{z}\right) E\left(w_{0}+g(w)\right) d t
\end{aligned}
$$




$$
\begin{aligned}
& =\int_{0}^{\infty} \int_{0}^{\infty} \frac{-1}{\mu} \frac{d}{d t}\left(e^{-\mu t \theta} \varpi_{z}(\theta)\right) E^{-1} \mathcal{C}\left(t^{z}\right) E\left(w_{0}+g(w)\right) d \theta d t \\
& =\int_{0}^{\infty} e^{-\mu t} \int_{0}^{\infty} E^{-1} \varpi_{z}(\theta) \mathcal{C}\left(\frac{t^{z}}{\theta^{z}}\right) E\left(w_{0}+g(w)\right) d \theta d t \\
& =\int_{0}^{\infty} e^{-\mu t} \int_{0}^{\infty} E^{-1} \frac{1}{z \theta^{(1+1 / z)}} \varpi_{z}\left(\theta^{-1 / z}\right) \mathcal{C}\left(t^{z} \theta\right) E\left(w_{0}+g(w)\right) d \theta d t \\
& =\int_{0}^{\infty} e^{-\mu t} \int_{0}^{\infty} E^{-1} \vartheta_{z}(\theta) \mathcal{C}\left(t^{z} \theta\right) E\left(w_{0}+g(w)\right) d \theta d t \\
& =\int_{0}^{\infty} e^{-\mu t}\left[\mathcal{S}_{E}(t) E\left(w_{0}+g(w)\right)\right] d t \\
& =\mathcal{L}\left[\mathcal{S}_{E}(t) E\left(w_{0}+g(w)\right)\right](\mu),
\end{aligned}
$$

where $\mathcal{L}$ stands for the Laplace transform. Furthermore, since $\mathcal{L}\left[g_{1}(t)\right](\mu)=\mu^{-1}$, by the Laplace convolution theorem, one can get

$$
\begin{aligned}
\mu^{-1} \mu^{z-1} \int_{0}^{\infty} E^{-1} e^{-\mu^{z} y} \mathcal{C}(y) E w_{1} d y & =\mathcal{L}\left[g_{1}(x)\right](\mu) \cdot \mathcal{L}\left[\mathcal{S}_{E}(t) E w_{1}\right](\mu) \\
& =\mathcal{L}\left[\left(g_{1} * \mathcal{S}_{E}\right)(t) E w_{1}\right](\mu)
\end{aligned}
$$

Similarly

$$
\begin{aligned}
\int_{0}^{\infty} & E^{-1} e^{-\mu^{z} y} \mathcal{S}(y) m(\mu) d y \\
\quad= & \int_{0}^{\infty} e^{-\mu t}\left[z \int_{0}^{t} \int_{0}^{\infty} E^{-1} \varpi_{z}(\theta) \mathcal{S}\left(\frac{(t-y)^{z}}{\theta^{z}}\right) a(y) J(y, w(y)) \frac{(t-y)^{z-1}}{\theta^{z}} d \theta d y\right] d t \\
= & \mathcal{L}\left[z \int_{0}^{t}(t-y)^{z-1} \int_{0}^{\infty} E^{-1} \varpi_{z}(\theta) \mathcal{S}\left(\frac{(t-y)^{z}}{\theta^{z}}\right) a(y) J(y, w(y)) \frac{1}{\theta^{z}} d \theta d y\right](\mu) \\
= & \mathcal{L}\left[\int_{0}^{t}(t-y)^{z-1} \mathcal{P}_{E}(t-y) a(y) J(y, w(y)) d y\right](\mu) .
\end{aligned}
$$

And also similarly

$$
\int_{0}^{\infty} E^{-1} e^{-\mu^{z} y} \mathcal{S}(y) n(\mu) d y=\mathcal{L}\left[\int_{0}^{t}(t-y)^{z-1} \mathcal{P}_{E}(t-y) K(y) v(y) d y\right](\mu) .
$$

Together with Eqs. (7), (8), (9) and (10), we get the desired result. Thus one accomplishes the proof.

Definition 3.1 For any $v \in L^{p}(\Omega, V)(1 \leq p \leq \infty)$, if there exists $w \in C\left(\Omega, U_{\alpha}\right)$ that satisfies for $t \in \Omega$

$$
\begin{aligned}
w(t)= & \mathcal{S}_{E}(t) E\left(w_{0}+g(w)\right)+\chi_{E}(t) E w_{1}+\int_{0}^{t}(t-y)^{z-1} \mathcal{P}_{E}(t-y) a(y) J(y, w(y)) d y \\
& +\int_{0}^{t}(t-y)^{z-1} \mathcal{P}_{E}(t-y) K(y) v(y) d y,
\end{aligned}
$$

then the system (1) is said to have a mild solution with respect to $v$ on $[0, S]$. 
Lemma 3.2 There exist an operator $\mathcal{S}_{E}(\cdot), \chi_{E}(\cdot)$ and $\mathcal{P}_{E}(\cdot)$ endowed with the following qualities:

(i) For any fixed $t \geq 0, \mathcal{S}_{E}(t), \chi_{E}(t)$ and $\mathcal{P}_{E}(t)$ are linear and bounded operators, i.e., for any $w \in U$, it follows that

$$
\begin{aligned}
\left\|\mathcal{S}_{E}(t) w\right\| & \leq M\left\|E^{-1}\right\|\|w\|, \quad\left\|\chi_{E}(t) w\right\| \leq M t\left\|E^{-1}\right\|\|w\|, \\
\left\|\mathcal{P}_{E}(t) w\right\| & \leq \frac{M\left\|E^{-1}\right\|}{\Gamma(2 z)} t^{z}\|w\| .
\end{aligned}
$$

$\left\{\mathcal{S}_{E}(t)\right\}_{t \geq 0},\left\{\chi_{E}(t)\right\}_{t \geq 0}$ and $\left\{\mathcal{P}_{E}(t)\right\}_{t \geq 0}$ are strongly continuous.

(ii) $\left\{\mathcal{S}_{E}(t), t \geq 0\right\},\left\{\chi_{E}(t), t \geq 0\right\}$ and $\left\{\mathcal{P}_{E}(t), t \geq 0\right\}$, are strongly continuous.

(iii) For every $t>0, \mathcal{S}_{E}(t), \chi_{E}(t)$ and $\mathcal{P}_{E}(t)$ are also compact operators if $S(t)$ is compact.

(iv) For any $w \in U, \beta \in\left(0, \frac{1}{2}\right)$ and $\alpha \in\left(0, \frac{1}{2}\right)$

$$
\begin{aligned}
& G_{b} \mathcal{P}_{E}(t) w=G_{b}^{1-\beta} \mathcal{P}_{E}(t) G_{b}^{\beta} w, \quad t \in \Omega, \\
& \left\|G_{b}^{\alpha} \mathcal{P}_{E}(t)\right\| \leq C_{\alpha}\left\|E^{-1}\right\|\left(\frac{1}{\Gamma(z)}+\frac{1}{\Gamma(2 z)} t^{z}\right)\|w\|, \quad 0<t \leq S .
\end{aligned}
$$

(v) For any fixed $t \geq 0$ and $w \in U^{\alpha},\left\|\mathcal{S}_{E}(t) w\right\|_{\alpha} \leq M\left\|E^{-1}\right\|\|w\|_{\alpha}$, $\left\|\chi_{E}(t) w\right\|_{\alpha} \leq M t\left\|E^{-1}\right\|\|w\|_{\alpha}$ and $\left\|\mathcal{P}_{E}(t) w\right\|_{\alpha} \leq \frac{M\left\|E^{-1}\right\|}{\Gamma(2 z)} t^{z}\|w\|_{\alpha}$.

Proof (i) For any fixed $t \geq 0$. Since $E^{-1}$ and $\mathcal{C}(t)$ are linear operators, we can easily see that $\mathcal{S}_{E}(t), \chi_{E}(t)$ and $\mathcal{P}_{E}(t)$ are also linear operators.

From [27], for any $w \in U$ and fixed $t \geq 0$,

$$
\left\|\mathcal{S}_{E}(t) w\right\| \leq \int_{0}^{\infty} \vartheta_{z}(\theta)\left\|E^{-1}\right\|\left\|\mathcal{C}\left(t^{z} \theta\right) w\right\| d \theta \leq M\left\|E^{-1}\right\|\|w\|
$$

from which one deduces immediately that

$$
\left\|\chi_{E}(t) w\right\| \leq M t\left\|E^{-1}\right\|\|w\|
$$

and we have

$$
\begin{aligned}
\left\|\mathcal{P}_{E}(t) w\right\| & \leq \int_{0}^{\infty} z \theta \vartheta_{z}(\theta)\left\|E^{-1}\right\|\left\|\mathcal{S}\left(t^{z} \theta\right) w\right\| d \theta \\
& \leq \int_{0}^{\infty} z \theta \vartheta_{z}(\theta) \int_{0}^{t^{z} \theta}\|\mathcal{C}(\mathcal{T}) w\| d \mathcal{T} d \theta \\
& \leq M z\left\|E^{-1}\right\|\|w\| t^{z} \int_{0}^{\infty} \theta^{2} \vartheta_{z}(\theta) d \theta=\frac{M\left\|E^{-1}\right\|}{\Gamma(2 z)}\|w\| t^{z}
\end{aligned}
$$

(ii) For every $w \in U$, since $\{C(t)\}_{t \in \mathbb{R}}$ is strongly continuous, i.e., for any $\varepsilon>0$ and $t, y \in \mathbb{R}$, there exists $\delta>0$ with $|t-y|<\delta$, such that $\|\mathcal{C}(t) w-\mathcal{C}(y) w\|<\varepsilon$, we thus get, for any $t^{\prime}, t^{\prime \prime} \geq 0$ with $\left|t^{\prime \prime}-t^{\prime}\right|<\delta$,

$$
\left\|\mathcal{S}_{E}\left(t^{\prime \prime}\right) w-\mathcal{S}_{E}\left(t^{\prime}\right) w\right\| \leq \int_{0}^{\infty} E^{-1} \vartheta_{z}(\theta)\left\|\left(\mathcal{C}\left(\left(t^{\prime \prime}\right)^{z} \theta\right)-\mathcal{C}\left(\left(t^{\prime}\right)^{z} \theta\right)\right) w\right\| d \theta<\varepsilon
$$


which means that $\left\|\mathcal{S}_{E}\left(t^{\prime \prime}\right) w-\mathcal{S}_{E}\left(t^{\prime}\right) w\right\| \rightarrow 0$ as $t^{\prime \prime} \rightarrow t^{\prime}$. Furthermore,

$$
\left\|\chi_{E}\left(t^{\prime \prime}\right) w-\chi_{E}\left(t^{\prime}\right) w\right\|=\left\|\int_{t^{\prime}}^{t^{\prime \prime}} \mathcal{C}_{z}(y) w d y\right\| \leq M\|w\|\left\|E^{-1}\right\|\left|t^{\prime \prime}-t^{\prime}\right| \rightarrow 0, \quad \text { as } t^{\prime \prime} \rightarrow t^{\prime},
$$

and finally we get

$$
\begin{aligned}
\left\|\mathcal{P}_{E}\left(t^{\prime \prime}\right) w-\mathcal{P}_{E}\left(t^{\prime}\right) w\right\| & \leq \int_{0}^{\infty} q \theta E^{-1} \vartheta_{z}(\theta)\left\|\left(\mathcal{S}\left(\left(t^{\prime \prime}\right)^{z} \theta\right)-\mathcal{S}\left(\left(t^{\prime}\right)^{z} \theta\right)\right) w\right\| d \theta \\
& \leq \frac{M}{\Gamma(2 z)}\|w\|\left\|E^{-1}\right\|\left|\left(t^{\prime \prime}\right)^{z}-\left(t^{\prime}\right)^{z}\right| \rightarrow 0, \quad \text { as } t^{\prime \prime} \rightarrow t^{\prime} .
\end{aligned}
$$

(iii) Already [38] $\mathcal{S}_{E}(t)$ and $\mathcal{P}_{E}(t)$ are compact. Now for $\chi_{E}(t)$ for each positive constant $k$, set $\{w \in V:\|w\| \leq k\}$. Then $V_{k}$ is clearly a bounded subset of $V$. We prove that $V(t):=$ $\left\{\int_{0}^{t} \int_{0}^{\infty} E^{-1} \vartheta_{z}(\theta) \mathcal{C}\left(y^{z} \theta\right) w d \theta d y, w \in V_{k}\right\}$ is relatively compact in $V$ for any positive constant $k$ and $t \geq 0$. From (i) we know $\chi_{1}(t): V \rightarrow V$ are also linear and bounded. So they map $V_{k}$ into a bounded subset of $V$. Then $V(t)=E^{-1} \chi_{1}(t)\left(V_{k}\right)$ is relatively compact in $V$ for any $K>0$ and $t \geq 0$ due to the compactness of $E^{-1}: V \rightarrow U$.

(iv) For any $w \in U, \beta \in\left(0, \frac{1}{2}\right), \alpha \in\left(0, \frac{1}{2}\right]$, we have

$$
\begin{aligned}
G_{b} \mathcal{P}_{E}(t) w & =z \int_{0}^{\infty} E^{-1} \theta \vartheta_{z}(\theta) G_{b} \mathcal{S}\left(t^{z} \theta\right) w d \theta \\
= & z \int_{0}^{\infty} E^{-1} \theta \vartheta_{z}(\theta) G_{b}^{1-\beta} \mathcal{S}\left(t^{z} \theta\right) G_{b}^{\beta} w d \theta \\
= & G_{b}^{1-\beta} \mathcal{P}_{E}(t) G_{b}^{\beta} w, \\
\left\|G_{b}^{\alpha} \mathcal{P}_{E}(t) w\right\| & =\left\|z \int_{0}^{\infty} E^{-1} \theta \vartheta_{z}(\theta) G_{b}^{\alpha} \mathcal{S}\left(t^{z} \theta\right) w d \theta\right\| \\
& \leq\|z\|\left\|E^{-1}\right\|\|w\| \int_{0}^{\infty} \theta \vartheta_{z}(\theta)\left\|G_{b}^{\alpha} \mathcal{S}\left(t^{z} \theta\right)\right\| d \theta \|,
\end{aligned}
$$

by Theorem 2.4

$$
\begin{aligned}
\left\|G_{b}^{\alpha} \mathcal{P}_{E}(t) w\right\| & \leq z\left\|E^{-1}\right\|\|w\| \int_{0}^{\infty} \theta \vartheta_{z}(\theta) C_{\alpha}\left(1+t^{z} \theta\right) d \theta \\
& \leq z C_{\alpha}\left\|E^{-1}\right\|\left(\int_{0}^{\infty} \theta \vartheta_{z}(\theta) d \theta+t^{z} \int_{0}^{\infty} \theta^{2} \vartheta_{z}(\theta) d \theta\right)\|w\| \\
& \leq C_{\alpha}\left\|E^{-1}\right\|\left(\frac{1}{\Gamma(z)}+\frac{1}{\Gamma(2 z)} t^{z}\right)\|w\| .
\end{aligned}
$$

(v) For fixed $t \geq 0$ and $w \in U^{\alpha}$

$$
\begin{aligned}
\left\|\mathcal{S}_{E}(t) w\right\|_{\alpha} & \leq \int_{0}^{\infty}\left\|E^{-1}\right\| \vartheta_{z}(\theta)\left\|G_{b}^{\alpha} \mathcal{C}\left(t^{z} \theta\right) w\right\| d \theta \\
& \leq \int_{0}^{\infty}\left\|E^{-1}\right\| \vartheta_{z}(\theta)\left\|\mathcal{C}\left(t^{z} \theta\right)\right\|\left\|G_{b}^{\alpha} w\right\| d \theta \\
& \leq M\left\|E^{-1}\right\| \int_{0}^{\infty} \vartheta_{z}(\theta)\left\|G_{b}^{\alpha} w\right\| d \theta \\
& \leq M\left\|E^{-1}\right\|\|w\|_{\alpha} .
\end{aligned}
$$


Now

$$
\begin{aligned}
\left\|\chi_{E}(t) w\right\|_{\alpha} & \leq \int_{0}^{t} \int_{0}^{\infty}\left\|E^{-1}\right\| \vartheta_{z}(\theta)\left\|G_{b}^{\alpha} \mathcal{C}\left(y^{z} \theta\right) w\right\| d \theta d y \\
& \leq \int_{0}^{t} \int_{0}^{\infty}\left\|E^{-1}\right\| \vartheta_{z}(\theta)\left\|\mathcal{C}\left(y^{z} \theta\right)\right\|\left\|G_{b}^{\alpha} w\right\| d \theta d y \\
& \leq M\left\|E^{-1}\right\| \int_{o}^{t} \int_{0}^{\infty} \vartheta_{z}(\theta)\left\|G_{b}^{\alpha} w\right\| d \theta d y \\
& \leq M t\left\|E^{-1}\right\|\|w\|_{\alpha}
\end{aligned}
$$

and similarly we have

$$
\begin{aligned}
\left\|\mathcal{P}_{E}(t) w\right\|_{\alpha} & \leq z \int_{0}^{\infty}\left\|E^{-1}\right\| \theta \vartheta_{z}(\theta)\left\|G_{b}^{\alpha} \mathcal{S}\left(t^{z} \theta\right) w\right\| d \theta \\
& \leq z \int_{0}^{\infty}\left\|E^{-1}\right\| \theta \vartheta_{z}(\theta)\left\|G_{b}^{\alpha} \int_{0}^{t^{z} \theta} \mathcal{C}(\mathcal{T}) d \mathcal{T} w\right\| d \theta \\
& \leq z \int_{0}^{\infty}\left\|E^{-1}\right\| \theta \vartheta_{z}(\theta)\left\|\int_{0}^{t^{z} \theta} \mathcal{C}(\mathcal{T}) d \mathcal{T}\right\|\left\|G_{b}^{\alpha} w\right\| d \theta \\
& \leq z M\left\|E^{-1}\right\| t^{z} \int_{0}^{\infty} \theta \vartheta_{z}(\theta)\left\|G_{b}^{\alpha} w\right\| d \theta \\
& \leq \frac{M\left\|E^{-1}\right\|}{\Gamma(2 z)} t^{z}\|w\|_{\alpha} .
\end{aligned}
$$

Thus one accomplishes the proof.

Theorem 3.3 Assume that (H1), (H2), (H3) and (H4) hold. Then, for each $v \in P_{a d}$, the system (1) is uniquely and mildly solvable on the closed interval $[0, S]$ with respect to $v$.

Proof (I) Local existence.

Let $S_{1} \leq S$ and $C_{0, S_{1}, \alpha}:=C\left(\left[0, S_{1}\right], U^{\alpha}\right)$ be provided with the usual sup-norm and set

$$
B\left(\lambda, S_{1}\right)=\left\{h \in C_{0, S_{1}, \alpha}: \max _{y \in\left[0, S_{1}\right]}\left\|h(y)-\left(w_{0}+g(w)\right)-y w_{1}\right\| \leq \lambda\right\} ; \quad \lambda \geq 0 .
$$

Then $B\left(\lambda, S_{1}\right) \subseteq C_{0, S_{1}, \alpha}$ is a closed convex subset of $C_{0, S_{1}, \alpha}$. In relation to (H1)(i) and (H1)(ii), it can be easily seen that $J(y, h(y))$ is a measurable function on $\left[0, S_{1}\right]$. Let $h \in B\left(\lambda, S_{1}\right)$, there exists a constant $\sigma^{*}:=\left\|G_{b}^{\alpha-\beta}\right\|\left\|w_{0}\right\|+\left\|G_{b}^{\alpha-\beta}\right\|\left\|w_{1}\right\| S_{1}+\lambda$ such that

$$
\|h\|_{0, S_{1}, \alpha} \leq \sigma^{*} .
$$

Using (H1)(iii), for $t \in\left[0, S_{1}\right]$, we have

$$
\|J(y, h(y))\| \leq C_{J}\left(1+\|h(y)\|_{0, S_{1}, \alpha}\right) \leq C_{J}\left(1+\sigma^{*}\right) \equiv K \quad \text { for } t \in\left[0, S_{1}\right]
$$


By the use of Lemma 3.2(iv), the Hölder inequality and (12) we obtain

$$
\begin{aligned}
& \int_{0}^{t}(t-y)^{z-1}\left\|\mathcal{P}_{E}(t-y) a(y) J(y, h(y))\right\|_{\alpha} d y \\
& \quad \leq K C_{\alpha}\left\|E^{-1}\right\|\left(\frac{1}{\Gamma(z)} S_{1}^{z-\frac{1}{p_{1}}}+\frac{1}{\Gamma(2 z)} S_{1}^{2 z-\frac{1}{p_{1}}}\right)\|a\|_{L^{P_{1}[0, b]}}
\end{aligned}
$$

Thus, $\left|(t-y)^{z-1} \mathcal{P}_{E}(t-y) a(y) J(y, h(y))\right|$ is Bochner integrable with respect to $y \in[0, t]$ for all $t \in\left[0, S_{1}\right]$.

On the other hand, by Lemma 3.2(iv) and the Hölder inequality, we see that

$$
\int_{0}^{t}(t-y)^{z-1}\left\|\mathcal{P}_{E}(t-y) K(y) v(y)\right\|_{\alpha} d y \leq C_{\alpha}\left\|E^{-1}\right\|\left(\frac{1}{\Gamma(z)} S_{1}^{z-\frac{1}{p}}+\frac{1}{\Gamma(2 z)} S_{1}^{2 z-\frac{1}{p}}\right)\|K v\|_{L^{P} \Omega}
$$

Thus $(t-y)^{z-1} \mathcal{P}_{E}(t-y) K(y) v(y)$ is also Bochner integrable with respect to $y \in[0, t]$ for all $t \in\left[0, S_{1}\right]$. Now for $0 \leq t \leq S_{1}$, we define $\mathcal{F}: B\left(\lambda, S_{1}\right) \rightarrow C_{0, S_{1}, \alpha}$ as follows:

$$
\begin{aligned}
(\mathcal{F} h)(t)= & \mathcal{S}_{E}(t) E\left(w_{0}+g(w)\right)+\chi_{E}(t) E w_{1}+\int_{0}^{t}(t-y)^{z-1} \mathcal{P}_{E}(t-y) a(y) J(y, h(y)) d y \\
& +\int_{0}^{t}(t-y)^{z-1} \mathcal{P}_{E}(t-y) K(y) v(y) d y .
\end{aligned}
$$

By the properties of $\mathcal{S}_{z}(\cdot), \chi_{z}(\cdot), \mathcal{P}_{z}(\cdot)$ and $(\mathrm{H} 1)$, one can prove that $\mathcal{F}$ is a contraction map on $B\left(\lambda, S_{1}\right)$ with suitable chosen $S_{1}>0$. Indeed, for $t \in\left[0, S_{1}\right]$, the following inequality can easily be obtained:

$$
\begin{aligned}
&\left\|(\mathcal{F} h)(t)-\left(w_{0}+g(w)\right)-t w_{1}\right\|_{\alpha} \\
& \leq\left\|\mathcal{S}_{E}(t) E\left(w_{0}+g(w)\right)-\left(w_{0}+g(w)\right)\right\|_{\alpha}+\left\|\chi_{E}(t) E w_{1}-t w_{1}\right\|_{\alpha} \\
&+\int_{0}^{t}(t-y)^{z-1}\left\|\mathcal{P}_{E}(t-y) a(y) J(y, h(y))\right\|_{\alpha} d y \\
&+\int_{0}^{t}(t-y)^{z-1}\left\|\mathcal{P}_{E}(t-y) K(y) v(y)\right\|_{\alpha} d y \\
& \leq\left\|G^{\alpha-\mu}\right\|\left\|\mathcal{S}_{E}(t) E G^{\mu}\left(w_{0}+g(w)\right)-G^{\mu}\left(w_{0}+g(w)\right)\right\| \\
&+\left\|G^{\alpha-\mu}\right\|\left\|\chi_{E}(t) E G^{\mu} w_{1}-G^{\mu} t w_{1}\right\| \\
&+K C_{\alpha}\left\|E^{-1}\right\|\left(\frac{1}{\Gamma(z)} S^{z-\frac{1}{p_{1}}}+\frac{1}{\Gamma(2 z)} S_{1}^{2 z-\frac{1}{p_{1}}}\right)\|a\|_{L^{P_{1}}[0, b]} \\
&+C_{\alpha}\left\|E^{-1}\right\|\left(\frac{1}{\Gamma(z)} S_{1}^{z-\frac{1}{p}}+\frac{1}{\Gamma(2 z)} S_{1}^{2 z-\frac{1}{p}}\right)\|K v\|_{L^{P} \Omega} .
\end{aligned}
$$

Since $\left\{\mathcal{S}_{E}(t)\right\}_{t \geq 0}$ and $\left\{\chi_{E}(t)\right\}_{t \geq 0}$ are strongly continuous operators in $U$, we can select $\varepsilon=\frac{\lambda}{3}$ such that

$$
\begin{aligned}
& \left\|\mathcal{S}_{E}(t) E G^{\mu}\left(w_{0}+g(w)\right)-G^{\mu}\left(w_{0}+g(w)\right)\right\| \leq \frac{\lambda}{3\left\|G^{\alpha-\mu}\right\|} \quad \text { and } \\
& \left\|\chi_{E}(t) E G^{\mu} w_{1}-G^{\mu} t w_{1}\right\| \leq \frac{\lambda}{3\left\|G^{\alpha-\mu}\right\|}
\end{aligned}
$$


Let

$$
S_{11}=\min \left\{\frac{\lambda}{3},\left(\frac{\Gamma(z) \Gamma(2 z)}{3 C_{\alpha}\left\|E^{-1}\right\|\left[(\Gamma(z)+\Gamma(2 z))\left[K\|a\|_{L^{P_{1}}[0, b]}+\|K v\|_{L^{p} \Omega}\right]\right]}\right)^{\frac{p}{2 z p-1}}\right\}
$$

Then, for all $t \in\left[0, S_{11}\right]$, we obtain from (13) and (14)

$$
\left\|(\mathcal{F} h)(t)-\left(w_{0}+g(w)\right)-t w_{1}\right\| \leq \lambda .
$$

Hence

$$
B\left(B\left(\lambda, S_{1}\right)\right) \subseteq B\left(\lambda, S_{1}\right)
$$

Let $h_{1}, h_{2} \in B\left(\lambda, S_{1}\right)$ and $\left\|h_{1}\right\|_{0, S_{1}, \alpha},\left\|h_{2}\right\|_{0, S_{1}, \alpha} \leq \sigma^{*}$. Denote

$$
P^{1}(y)=J\left(y, h_{1}(y)\right) \text { and } P^{2}(y)=J\left(y, h_{2}(y)\right) .
$$

For $t \in\left[0, S_{1}\right]$, using Lemma 3.2(iv) and (H1)(iii), we obtain

$$
\begin{aligned}
\left\|\left(\mathcal{F} h_{1}\right)(t)-\left(\mathcal{F} h_{2}\right)(t)\right\|_{\alpha} \leq & \int_{0}^{t}(t-y)^{z-1}\left\|\mathcal{P}_{E}(t-y) a(y)\left(P^{1}(y)-P^{2}(y)\right)\right\|_{\alpha} d y \\
\leq & C_{\alpha}\left\|E^{-1}\right\| L_{J}\left(\sigma^{*}\right)\left(\frac{1}{\Gamma(z)} \int_{0}^{t}(t-y)^{z-1} a(y)\left\|h_{1}(y)-h_{2}(y)\right\|_{\alpha} d y\right. \\
& \left.+\frac{1}{\Gamma(2 z)} \int_{0}^{t}(t-y)^{2 z-1} a(y)\left\|h_{1}(y)-h_{2}(y)\right\|_{\alpha} d y\right),
\end{aligned}
$$

which implies that

$$
\begin{aligned}
& \left\|\left(\mathcal{F} h_{1}\right)(t)-\left(\mathcal{F} h_{2}\right)(t)\right\| \\
& \quad \leq C_{\alpha}\left\|E^{-1}\right\| L_{J}\left(\sigma^{*}\right)\left(\frac{1}{\Gamma(z)} t^{z-\frac{1}{p_{1}}}+\frac{1}{\Gamma(2 z)} t^{2 z-\frac{1}{p_{1}}}\right)\|a\|_{L^{p_{1}}[0, b]}\left\|h_{1}-h_{2}\right\|_{0, S_{1}, \alpha} .
\end{aligned}
$$

Thus

$$
\begin{aligned}
& \left\|\mathcal{F} h_{1}-\mathcal{F} h_{2}\right\|_{0, S_{1}, \alpha} \\
& \quad \leq C_{\alpha}\left\|E^{-1}\right\| L_{J}\left(\sigma^{*}\right)\left(\frac{1}{\Gamma(z)} S^{z-\frac{1}{p_{1}}}+\frac{1}{\Gamma(2 z)} S^{2 z-\frac{1}{p_{1}}}\right)\|a\|_{L^{p_{1}}[0, b]}\left\|h_{1}-h_{2}\right\|_{0, S_{1}, \alpha} .
\end{aligned}
$$

Let $S_{12}=\frac{1}{2}\left(\frac{\Gamma(z) \Gamma(2 z)}{C_{\alpha}\left\|E^{-1}\right\|\left(\Gamma(z)+\Gamma(2 z)\|a\|_{L} p_{1[0, b]} L_{J}\left(\sigma^{*}\right)\right.}\right)^{\frac{p_{1}}{2 z p_{1}-1}} ; S_{1}=\min \left\{S_{11}, S_{12}\right\}$. Then $\mathcal{F}$ is a contraction map on $B\left(\lambda, S_{1}\right)$. It follows from the contraction mapping principle that $\mathcal{F}$ has a unique fixed point $h \in B\left(\lambda, S_{1}\right)$, and the unique and mild solution of the system (1) is $h$ with respect to $v$ on the close interval $\left[0, S_{1}\right]$.

Proof (II) Global existence.

If a mild solution of the system (1) is $w$ with respect to $v$ on the closed interval [0,S], then $w$ satisfies (12). Using (H1)(iii), (H2)(ii) Lemma 3.2(iv) and the Hölder inequality, we 
obtain

$$
\begin{aligned}
\|w(t)\|_{\alpha} \leq & \left\|\mathcal{S}_{E}(t) E\left(w_{0}+g(w)\right)\right\|_{\alpha}+\left\|\chi_{E}(t) E w_{1}\right\|_{\alpha} \\
& +\int_{0}^{t}(t-y)^{z-1}\left\|\mathcal{P}_{E}(t-y) a(y) J(y, w(y))\right\|_{\alpha} d y \\
& +\int_{0}^{t}(t-y)^{z-1}\left\|\mathcal{P}_{E}(t-y) K(y) v(y)\right\|_{\alpha} d y \\
\leq & M\left\|E^{-1}\right\|\left\|G^{\alpha-\beta}\right\|\|E\|\left\|w_{0}\right\|+M\left\|E^{-1}\right\|\left\|G^{\alpha-\beta}\right\|\|E\|\|g(w)\| \\
& +N\left\|E^{-1}\right\|\left\|G^{\alpha-\beta}\right\|\|E\|\left\|w_{1}\right\| \\
& +C_{\alpha}\left\|E^{-1}\right\|\left(\frac{1}{\Gamma(z)} \int_{0}^{t}(t-y)^{z-1} a(y) C_{J}\left(1+\|w(y)\|_{0, S, \alpha}\right) d y\right. \\
& \left.+\frac{1}{\Gamma(2 z)} \int_{0}^{t}(t-y)^{2 z-1} a(y) C_{J}\left(1+\|w(y)\|_{0, S, \alpha}\right) d y\right) \\
& C_{\alpha}\left\|E^{-1}\right\|\left(\frac{1}{\Gamma(z)} \int_{0}^{t}(t-y)^{z-1}\|K(y) v(y)\| d y\right. \\
& \left.+\frac{1}{\Gamma(2 z)} \int_{0}^{t}(t-y)^{2 z-1}\|K(y) v(y)\| d y\right) \\
\leq & M\left\|G^{\alpha-\beta}\right\|\left\|w_{0}\right\|+M N_{g 1}\left\|G^{\alpha-\beta}\right\|\|w\|_{C}+M N_{g 2}+N\left\|G^{\alpha-\beta}\right\|\left\|w_{1}\right\| \\
& +C_{\alpha}\left\|E^{-1}\right\|\left(\frac{1}{\Gamma(z)} \int_{0}^{t}(t-y)^{z-1} a(y) C_{J}\left(1+\|w(y)\|_{0, S, \alpha}\right) d y\right. \\
& \left.+\frac{1}{\Gamma(2 z)} \int_{0}^{t}(t-y)^{2 z-1} a(y) C_{J}\left(1+\|w(y)\|_{0, S, \alpha}\right) d y\right) \\
& C_{\alpha}\left\|E^{-1}\right\|\left(\frac{1}{\Gamma(z)} \int_{0}^{t}(t-y)^{z-1}\|K(y) v(y)\| d y\right. \\
& \left.+\frac{1}{\Gamma(2 z)} \int_{0}^{t}(t-y)^{2 z-1}\|K(y) v(y)\| d y\right) \\
& +b+C_{\alpha} C_{J}\left\|E^{-1}\right\|\left(\frac{1}{\Gamma(z)} \int_{0}^{t}(t-y)^{z-1} a(y)\|w(y)\|_{0, S, \alpha} d y\right. \\
& \left.(t-y)^{2 z-1} a(y)\|w(y)\|_{0, S, \alpha} d y\right), \\
& \\
&
\end{aligned}
$$

where

$$
\begin{aligned}
a+b= & M\left\|G^{\alpha-\beta}\right\|\left\|w_{0}\right\|+M N_{g 1}\left\|G^{\alpha-\beta}\right\|\|w\|_{C}+M N_{g 2}+N\left\|G^{\alpha-\beta}\right\|\left\|w_{1}\right\| \\
& +C_{\alpha} C_{J}\left\|E^{-1}\right\|\left(\frac{1}{\Gamma(z)} S^{z-\frac{1}{p_{1}}}+\frac{1}{\Gamma(2 z)} S^{2 z-\frac{1}{p_{1}}}\right)\|a\|_{L^{p_{1}}[0, b]} \\
& +C_{\alpha}\left\|E^{-1}\right\|\left(\frac{1}{\Gamma(z)} S^{z-\frac{1}{p}}+\frac{1}{\Gamma(2 z)} S^{2 z-\frac{1}{p}}\right)\|K v\|_{L^{P} \Omega}
\end{aligned}
$$

where $N=M S$. By Lemma 2.2, there exists a constant $M^{*}>0$ such that

$$
\|w(t)\|_{\alpha} \leq M^{*}(a)+M^{*}(b)=M^{*}(c), \quad \text { for } t \in \Omega .
$$


Let

$$
S_{21}=S_{1}+S_{11}, \quad S_{22}=S_{1}+S_{12}, \quad \Delta S=\min \left\{S_{21}-S_{1}, S_{12}\right\}>0 .
$$

In the same way, it can be verified that a unique mild solution exists for the system (1) on $[0, \Delta S]$. In regard to the procedure mentioned above in each interval $[\Delta S, 2 \Delta S]$, $[2 \Delta S, 3 \Delta S], \ldots$ implementing the method of the above arguments, the existence of a mild solution can easily be obtained for the system (1).

\section{Continuous dependence}

In this section, we show that the mild solution of the system (1) shows a continuous dependence on the initial value with respect to the control term.

Theorem 4.1 Assume that $w_{0}^{1}, w_{0}^{2} \in \eta$ where $\eta$ is a bounded set. Let

$$
\begin{aligned}
& w^{1}\left(t, w_{0}^{1}+g\left(w^{1}\right), w_{1}^{1}, v\right) \\
& =\mathcal{S}_{E}(t) E\left(w_{0}^{1}+g\left(w^{1}\right)\right)+\chi_{E}(t) E w_{1}^{1}+\int_{0}^{t}(t-y)^{z-1} \mathcal{P}_{E}(t-y) a(y) J\left(y, w^{1}(y)\right) d y \\
& \quad+\int_{0}^{t}(t-y)^{z-1} \mathcal{P}_{E}(t-y) K(y) v(y) d y, \quad 0 \leq t \leq S,
\end{aligned}
$$

and

$$
\begin{aligned}
& w^{2}\left(t, w_{0}^{2}+g\left(w^{2}\right), w_{1}^{2}, u\right) \\
& =\mathcal{S}_{E}(t) E\left(w_{0}^{2}+g\left(w^{2}\right)\right)+\chi_{E}(t) E w_{1}^{2}+\int_{0}^{t}(t-y)^{z-1} \mathcal{P}_{E}(t-y) a(y) J\left(y, w^{2}(y)\right) d y \\
& \quad+\int_{0}^{t}(t-y)^{z-1} \mathcal{P}_{E}(t-y) K(y) u(y) d y, \quad 0 \leq t \leq S .
\end{aligned}
$$

Then there exists a constant $C^{*}$ such that

$$
\begin{aligned}
& \left\|w^{1}\left(t, w_{0}^{1}+g\left(w^{1}\right), w_{1}^{1}, v\right)-w^{2}\left(t, w_{0}^{2}+g\left(w^{2}\right), w_{1}^{2}, u\right)\right\| \\
& \quad \leq C^{*}\left(\left\|w_{0}^{1}-w_{0}^{2}\right\|+\left\|w^{1}-w^{2}\right\|_{C}+\left\|w_{1}^{1}-w_{1}^{2}\right\|+\|K v-K u\|_{L^{p} \Omega}\right), \quad t \in \Omega,
\end{aligned}
$$

where

$$
\begin{aligned}
C^{*}= & \max \left\{N\left\|G^{\alpha-\beta}\right\|, M L_{g}(\sigma)\left\|G^{\alpha-\beta}\right\|, M\left\|G^{\alpha-\beta}\right\|,\right. \\
& \left.C_{\alpha}\left\|E^{-1}\right\|\left(\frac{1}{\Gamma(z)} S^{z-\frac{1}{p}}+\frac{1}{\Gamma(2 z)} S^{2 z-\frac{1}{p}}\right)\right\}>0 .
\end{aligned}
$$

Proof Since $w_{0}^{1}, w_{0}^{2} \in \eta$, here $\eta$ is a bounded set in $U$, using Theorem 3.3(II), there exists a constant $\sigma>0$ such that $\left|w^{1}\right|,\left|w^{2}\right| \leq \sigma$. For $t \in \Omega$, by Lemma 3.2, (H1)(ii), (H2)(i) and the Hölder inequality we have

$$
\begin{aligned}
& \left\|w^{1}\left(t, w_{0}^{1}+g\left(w^{1}\right), w_{1}^{1}, v\right)-w^{2}\left(t, w_{0}^{2}+g\left(w^{2}\right), w_{1}^{2}, u\right)\right\|_{\alpha} \\
& \quad \leq\left\|\mathcal{S}_{E}(t) E\left(\left(w_{0}^{1}+g\left(w^{1}\right)\right)-\left(w_{0}^{2}+g\left(w^{2}\right)\right)\right)\right\|_{\alpha}
\end{aligned}
$$




$$
\begin{aligned}
& +\left\|\chi_{E}(t) E\left(w_{1}^{1}-w_{1}^{2}\right)\right\|_{\alpha} \\
& +\int_{0}^{t}(t-y)^{z-1}\left\|\mathcal{P}_{E}(t-y) a(y)\left(J\left(y, w^{1}(y)\right)-J\left(y, w^{2}(y)\right)\right)\right\|_{\alpha} d y \\
& +\int_{0}^{t}(t-y)^{z-1}\left\|\mathcal{P}_{E}(t-y)(K(y) v(y)-K(y) u(y))\right\|_{\alpha} d y \\
& \leq M\left\|E^{-1}\right\|\left\|G^{\alpha-\beta}\right\|\|E\|\left\|w_{0}^{1}-w_{0}^{2}\right\| \\
& +M\left\|E^{-1}\right\|\left\|G^{\alpha-\beta}\right\|\|E\|\left\|g\left(w^{1}\right)-g\left(w^{2}\right)\right\|+N\left\|E^{-1}\right\|\left\|G^{\alpha-\beta}\right\|\|E\|\left\|w_{1}^{1}-w_{1}^{2}\right\| \\
& +C_{\alpha}\left\|E^{-1}\right\|\left(\frac{1}{\Gamma(z)} S^{z-\frac{1}{p}}+\frac{1}{\Gamma(2 z)} S^{2 z-\frac{1}{p}}\right)\|K v-K u\|_{L^{P} \Omega} \\
& +C_{\alpha} L_{J}(\sigma)\left\|E^{-1}\right\|\left(\frac{1}{\Gamma(z)} \int_{0}^{t}(t-y)^{z-1} a(y)\left\|w^{1}(y)-w^{2}(y)\right\|_{\alpha} d y\right. \\
& \left.+\frac{1}{\Gamma(2 z)} \int_{0}^{t}(t-y)^{2 z-1} a(y)\left\|w^{1}(y)-w^{2}(y)\right\|_{\alpha} d y\right),
\end{aligned}
$$

which implies that

$$
\begin{aligned}
& \left\|w^{1}\left(t, w_{0}^{1}+g\left(w^{1}\right), w_{1}^{1}, v\right)-w^{2}\left(t, w_{0}^{2}+g\left(w^{2}\right), w_{1}^{2}, u\right)\right\| \\
& \leq M\left\|G^{\alpha-\beta}\right\|\left\|w_{0}^{1}-w_{0}^{2}\right\|+M L_{g}(\sigma)\left\|G^{\alpha-\beta}\right\|\left\|w^{1}-w^{2}\right\|_{C} \\
& \quad+N\left\|G^{\alpha-\beta}\right\|\left\|w_{1}^{1}-w_{1}^{2}\right\|+C_{\alpha}\left\|E^{-1}\right\|\left(\frac{1}{\Gamma(z)} S^{z-\frac{1}{p}}+\frac{1}{\Gamma(2 z)} S^{2 z-\frac{1}{p}}\right)\|K v-K u\|_{L^{P} \Omega} \\
& \quad+C_{\alpha} L_{J}(\sigma)\left\|E^{-1}\right\|\left(\frac{1}{\Gamma(z)} \int_{0}^{t}(t-y)^{z-1} a(y)\left\|w^{1}(y)-w^{2}(y)\right\|_{\alpha} d y\right. \\
& \left.\quad+\frac{1}{\Gamma(2 z)} \int_{0}^{t}(t-y)^{2 z-1} a(y)\left\|w^{1}(y)-w^{2}(y)\right\|_{\alpha} d y\right)
\end{aligned}
$$

and, using Lemma 2.2 again, we obtain

$$
\begin{aligned}
& \left\|w^{1}\left(t, w_{0}^{1}+g\left(w^{1}\right), w_{1}^{1}, v\right)-w^{2}\left(t, w_{0}^{2}+g\left(w^{2}\right), w_{1}^{2}, u\right)\right\| \\
& \quad \leq C^{*}\left(\left\|w_{0}^{1}-w_{0}^{2}\right\|+\left\|w^{1}-w^{2}\right\|_{C}+\left\|w_{1}^{1}-w_{1}^{2}\right\|+\|K v-K u\|_{L^{p} \Omega}\right), \quad t \in \Omega .
\end{aligned}
$$

Thus one accomplishes the proof.

\section{Optimal control}

In this section, we study the existence of optimal pairs for the fractional control system (1). Firstly, we notice the following Lagrange problem:

(P) find a control $v^{\circ} \in P_{a d}$ such that

$$
\mathcal{J}\left(v^{\circ}\right) \leq \mathcal{J}(v) \quad \text { for all } v \in P_{a d},
$$

where

$$
\mathcal{J}(v)=\int_{0}^{S} \mathcal{I}\left(t, w^{v}(t), v(t)\right) d t,
$$

$w^{v}$ illustrates the mild solution of the system (1) corresponding to the control $v \in P_{a d}$. 
For the existence of a solution for problem $(P)$, we put forward the following assumption:

(H5) (i) the functional $\mathcal{I}: \Omega \times U \times V \rightarrow R \cup\{\infty\}$ is Borel measurable;

(ii) $\mathcal{I}(t . \cdot . \cdot)$ is sequentially lower semi continuous on $U \times V$ for almost all $t \in \Omega$;

(iii) $\mathcal{I}(t, w, \varsigma$.$) is convex on \varsigma$ for each $w \in C_{0, S}, \varsigma \in U$ and almost all $t \in \Omega$;

(iv) there exist constant, $e \geq 0, j>0, \phi$ is nonnegative and $\phi \in L^{1}(\Omega, \mathbb{R})$ such that

$$
\mathcal{I}(t, \varsigma, v) \geq \phi(t)+e|\varsigma|+j\|v\|_{V}^{p}
$$

Now, we can show the following results on the existence of fractional optimal control for problem $(P)$.

We are in dire need of a relevant lemma to obtain the existence of optimal control.

Lemma 5.1 ([19]) Suppose $G$ has a compact resolvent. Then for some $p z(1-\alpha)>1$ the operator

$$
\mathcal{R}(l(\cdot))=\int_{0}^{\cdot}(\cdot-y)^{z-1} P_{E}(\cdot-y) l(y) d y, \quad l(\cdot) \in L^{p} \Omega
$$

is a compact operator from $L^{p}(\Omega, U)$ to $C\left(\Omega, U_{\alpha}\right)$.

Theorem 5.2 Make the assumption of Theorem 3.3 and (H5). If G has a compact resolvent then problem $(P)$ admits at least one optimal pair.

Proof If $\inf \left\{\mathcal{J}(v) \mid v \in P_{a d}\right\}=+\infty$, nothing can be proved.

So assume that

$$
\inf \left\{\mathcal{J}(v) \mid v \in P_{a d}\right\}=\varepsilon<+\infty \text {. }
$$

Using the assumption (H5), we have $\varepsilon>-\infty$. By the definition of an infimum, there exists a minimizing sequence feasible pair

$$
\begin{aligned}
& \left\{\left(w^{r}, v^{r}\right)\right\} \subset A_{a d} \\
& \quad:=\left\{(w, v) ; w \text { is a mild solution of the system (1) corresponding to } v \in P_{a d}\right\},
\end{aligned}
$$

such that $\mathcal{J}\left(w^{r}, v^{r}\right) \rightarrow \varepsilon$ as $r \rightarrow+\infty$. Since $\left\{v^{r}\right\} \subseteq P_{a d}, r=1,2, \ldots,\left\{v^{r}\right\}$ is a bounded subset of the separable reflexive Banach space $L^{p}(\Omega, V)$, there exist a subsequence (we denote it by $\left.\left\{v^{r}\right\}\right)$ and $v^{\circ} \in L^{p}(\Omega, V)$ such that

$$
v^{r} \rightarrow v^{\circ} \quad \text { in } L^{p}(\Omega, V) .
$$

Since $P_{a d}$ is closed and convex, owing to the Marzur lemma, we have $v^{\circ} \in P_{a d}$. It can be estimated that $w^{r} \in C_{0, S, \alpha}$ illustrates the corresponding sequence of the solution of the integral equation. We have

$$
\begin{aligned}
w^{r}(t)= & \mathcal{S}_{E}(t) E\left(w_{0}+g\left(w^{1}\right)\right)+\chi_{E}(t) E w_{1}+\int_{0}^{t}(t-y)^{z-1} \mathcal{P}_{E}(t-y) a(y) J\left(y, w^{r}(y)\right) d y \\
& +\int_{0}^{t}(t-y)^{z-1} \mathcal{P}_{E}(t-y) K(y) v^{r}(y) d y, \quad 0 \leq t \leq S .
\end{aligned}
$$


Due to Lemma 2.2 and 3.3(II) again, we can prove that there exists a $\sigma>0$ such that

$$
\left\|w^{r}\right\|_{0, S, \alpha} \leq \sigma \quad \text { where } r=0,1,2, \ldots
$$

where $w^{\circ}$ denotes the solution corresponding to $v^{\circ}$, that is,

$$
\begin{aligned}
w^{\circ}(t)= & \mathcal{S}_{E}(t) E\left(w_{0}+g\left(w^{\circ}\right)\right)+\chi_{E}(t) E w_{1}+\int_{0}^{t}(t-y)^{z-1} \mathcal{P}_{E}(t-y) a(y) J\left(y, w^{\circ}(y)\right) d y \\
& +\int_{0}^{t}(t-y)^{z-1} \mathcal{P}_{E}(t-y) K(y) v^{\circ}(y) d y, \quad 0 \leq t \leq S .
\end{aligned}
$$

Hence, for $t \in \Omega$ by condition (H1)(ii), (H2)(i) Lemma 3.2(i), (iv) and the Hölder inequality, we obtain the following inequalities after an elementary calculation:

$$
\begin{aligned}
\| w^{r}(t) & -w^{\circ}(t) \|_{\alpha} \\
\leq & \| \mathcal{S}_{E}(t) E\left(\left(g\left(w^{r}\right)-g\left(w^{\circ}\right)\right) \|_{\alpha}\right. \\
& +\left\|\int_{0}^{t}(t-y)^{z-1} \mathcal{P}_{E}(t-y)\left[J\left(y, w^{r}(y)\right)-J\left(y, w^{\circ}(y)\right)\right] d y\right\|_{\alpha} \\
& +\left\|\int_{0}^{t}(t-y)^{z-1} \mathcal{P}_{E}(t-y)\left[K(y) v^{r}(y)-K(y) v^{\circ}(y)\right] d y\right\|_{\alpha} \\
\leq & M L_{g}(\sigma)\left\|w^{r}-w^{\circ}\right\|_{C}+\left\|\mathcal{R}\left(K(y) v^{r}(y)\right)-\mathcal{R}\left(K(y) v^{\circ}(y)\right)\right\|_{\alpha} d y \\
& +C_{\alpha} L_{J}(\sigma)\left\|E^{-1}\right\|\left(\frac{1}{\Gamma(z)} \int_{0}^{t}(t-y)^{z-1} a(y)\left\|w^{r}(y)-w^{\circ}(y)\right\|_{\alpha} d y\right. \\
& \left.+\frac{1}{\Gamma(2 z)} \int_{0}^{t}(t-y)^{2 z-1} a(y)\left\|w^{r}(y)-w^{\circ}(y)\right\|_{\alpha} d y\right) \\
:= & \xi_{r}^{(1)}+\xi_{r}^{(2)}+\xi_{r}^{(3)} .
\end{aligned}
$$

By applying Lemma 5.1 we have $\left\|\mathcal{R}\left(K(y) v^{r}(y)\right)-\mathcal{R}\left(K(y) v^{\circ}(y)\right)\right\|_{C\left(\Omega, U_{\alpha}\right)} \rightarrow 0$ as $r \rightarrow \infty$, which implies that $\xi_{r}^{(2)} \rightarrow 0$ as $r \rightarrow \infty$. Moreover, we have

$$
\begin{aligned}
\| w^{r}(t) & -w^{\circ}(t) \| \\
\leq & \left\|\xi_{r}^{(2)}\right\|_{\alpha}+M L_{g}(\sigma)\left\|w^{r}-w^{\circ}\right\|_{C} \\
& +C_{\alpha} L_{J}(\sigma)\left\|E^{-1}\right\|\left(\frac{1}{\Gamma(z)} \int_{0}^{t}(t-y)^{z-1} a(y)\left\|w^{r}(y)-w^{\circ}(y)\right\|_{\alpha} d y\right. \\
& \left.+\frac{1}{\Gamma(2 z)} \int_{0}^{t}(t-y)^{2 z-1} a(y)\left\|w^{r}(y)-w^{\circ}(y)\right\|_{\alpha} d y\right) .
\end{aligned}
$$

By virtue of the Gronwall inequality again, there exists $M^{*}>0$ such that

$$
\left\|w^{r}(t)-w^{\circ}(t)\right\|_{\alpha} \leq M^{*}\left\|\xi_{r}^{(2)}\right\|_{\alpha}
$$

which yields

$$
w^{r} \rightarrow w^{\circ} \quad \text { in } C_{0, S, \alpha} \text { as } r \rightarrow \infty .
$$


Note that assumption (H5) implies Balder's assumption. Hence, by Balder's theorem, we can jump to the conclusion that

$$
(w, v) \rightarrow \int_{0}^{S} \mathcal{I}(t, w(t), v(t)) d t
$$

is sequentially lower semi continuous in the weak topology of $L^{p}(\Omega, v) \subset L^{1}(\Omega, V)$, and the strong topology of $L^{1}\left(\Omega, U_{\alpha}\right)$. Hence, $\Omega$ is weakly lower semi continuous on $L^{p}(\Omega, V)$, and since, by (H5)(iv), $\Omega>-\infty, \Omega$ gets its infimum at $\nu_{0} \in P_{a d}$, i.e.,

$$
\begin{aligned}
\varepsilon & =\lim _{r \rightarrow \infty} \int_{0}^{S} \mathcal{I}\left(t, w^{r}(t), v^{r}(t)\right) d t \\
& \geq \int_{0}^{S} \mathcal{I}\left(t, w^{\circ}(t), v^{\circ}(t)\right) d t \\
& =\mathcal{J}(v \circ) \\
& \geq \varepsilon .
\end{aligned}
$$

Thus one accomplishes the proof.

We proceed to illustrate an example to show our results.

Example 5.3 Notice the following problem:

$$
\left\{\begin{array}{l}
{ }_{0}^{c} D_{t}^{\gamma} E w(t, s)-\Delta w(t, s) \\
\quad=a(y, t) w(t)(s)+\int_{\Sigma} L(s, y) v(y, t) d y, \quad y \in \Sigma y \leq t, 0<t \leq S \\
w(t, s)=0, \quad s \in \partial \Sigma, t \in \Omega, \\
w(0, s)-\sum_{i=1}^{n} \int_{\Sigma} m(\xi, s) w\left(t_{i}, \xi\right) d \xi=0, \quad w^{\prime}(0, s)=0, \quad s \in \Sigma
\end{array}\right.
$$

with the cost function

$$
\mathcal{I}(v(s, t))=\int_{0}^{S}\left(\int_{\Sigma}\left|w^{v}(t, s)\right|^{P} d s+\int_{\Sigma}|v(s, t)|^{P} d s\right) d t
$$

where $\Sigma \subset \mathbb{R}^{N}$ is a bounded domain, $\partial \Sigma \in C^{N}, \Delta$ is Laplace operator, $v \in L^{p}(\Omega \times \Sigma, \mathbb{R})$, $h \in L^{1}([0, S], \mathbb{R})$ and $K: \bar{\Sigma} \times \bar{\Sigma} \rightarrow R$ is continuous. $m(\xi, s): \Sigma \times \Sigma \rightarrow U$ is an $L^{P}$-Lebesgue integrable function.

Define

$$
\begin{aligned}
& U=V=L^{p}(\Omega \times \Sigma, \mathbb{R}), \quad D\left(-G E^{-1}\right)=\left\{W^{2, N}(\Sigma) \cap W_{0}^{1, N}(\Sigma)\right\} \\
& G w=-\Delta w \quad \text { for } w \in D\left(-G E^{-1}\right),
\end{aligned}
$$

for $w \in D\left(G E^{-1}\right)$. Then $-G E^{-1}$ can generate a strong continuous cosine family $\{\mathcal{C}(t), t \geq 0\}$ on $U$. Similarly, the fractional power operator $G^{\alpha}$ and the fractional power space $U^{\alpha}$ are introduced. The controls are functions $v: \mathcal{C} w(\Sigma) \rightarrow \mathbb{R}$, such that $v \in L^{p}(\mathcal{C} w(\Sigma))$. It can be claimed that $t \rightarrow v(\cdot, t)$ going from $\Omega$ into $V$ is measurable. Set

$$
P(t)=\left\{v \in V \mid\|v\|_{V} \leq \chi\right\},
$$


where $\chi \in L^{p}\left(\Omega, \mathbb{R}^{+}\right)$. We assume the admissible controls $P_{a d}$ to be all $v \in L^{p}(\mathcal{C} w(\Sigma))$ such that $\|v(\cdot, t)\|_{L^{p}(\Sigma)} \leq \chi(t)$, a.e. Let $\frac{1}{2}>\alpha>\frac{3}{7}$ and $p>\frac{1}{z(1-\alpha)}$. One can obtain the insertion relative to $U^{\alpha} \hookrightarrow C^{\prime}(\Sigma)$.

Define $w(t)(s)=w(t, s)$,

$$
K(t) v(t)(s)=\int_{\Sigma} L(s, y) v(y, t) d \xi
$$

and

$$
a(t) J(t, w(t))(s)=a(y, t) w(t)(s) .
$$

Taking $\gamma=\frac{3}{2}$, we have $z=\frac{3}{4}$. Let $g: C(\Omega, U) \rightarrow U$ be given by $g(w)(s)=\sum_{i=0}^{n} L_{g} w\left(t_{i}\right)(s)$ with $L_{g} v(s)=\int_{\Sigma} m(\xi, s) v(\xi) d \xi$ for $v \in U, s \in \Sigma$ (denoting that $L_{g}: U \rightarrow U$ is completely continuous). Thus the assumption in (H5) holds. We have

$$
\mathcal{I}\left(t, w^{v}(t), w^{v}(t), v(t)\right)(s)=\int_{\Sigma}\left|w^{v}(t, s)\right|^{P} d s+\int_{\Sigma}|v(t, s)|^{p} d s .
$$

We can write the problem (15) as

$$
\left\{\begin{array}{l}
{ }_{0}^{c} D_{t}^{\gamma} E w(t)+G w(t)=a(t) J(t, w(t))+K(t) v(t), \quad t \in \Omega \\
w(0)=w_{0}+g(w), \quad w^{\prime}(0)=w_{1} .
\end{array}\right.
$$

with the cost function

$$
\mathcal{J}(v)=\int_{0}^{S} \mathcal{I}\left(t, w^{v}(t), v(t)\right) d t
$$

Evidently, it confirms all the assumptions made in Theorem 5.2 that our findings can be extended to Problem (15). Thus, problem (15) admits at least one optimal pairs.

\section{Conclusion}

Our manuscript is mainly focused on optimal control for fractional differential evolution equations of order $1<\gamma<2$. By using the results on fractional calculus, $\alpha$-norms of cosine and sine families on operators, an adequate definition of $\alpha$-mild solutions is obtained and the existence, uniqueness and continuous dependence of $\alpha$-mild solutions for the presented control system are also established. The existence of optimal pairs of a nonlocal fractional evolution system is also demonstrated under the construction of the Lagrange problem. Then we develop our conclusions with a view on nonlocal conditions. Lastly, we present theoretical applications to support the validity of the study. In the future, we want to find controllability results for fuzzy fractional differential equations; we hope this will open new ways for researchers. 
Availability of data and materials

Not applicable.

\section{Competing interests}

The authors declare that they have no competing interests.

\section{Authors' contributions}

All authors contributed equally to the writing of this paper. All authors read and approved the final manuscript.

\section{Author details}

${ }^{1}$ Faculty of Mathematics and Computational Science, Xiangtan University, Hunan, 411105, China. ${ }^{2}$ Department of Mathematics and Statistics, University of Lahore, Sargodha, Pakistan. ${ }^{3}$ Department of Mathematics, Faculty of Science, University of Ha'il, Ha'il, 81481, Saudi Arabia. ${ }^{4}$ Department of Mathematics, Faculty of Science, Mansoura University, Mansoura, Egypt.

\section{Publisher's Note}

Springer Nature remains neutral with regard to jurisdictional claims in published maps and institutional affiliations.

\section{Received: 9 September 2020 Accepted: 18 February 2021 Published online: 27 February 2021}

\section{References}

1. Podlubny, I.: Fractional Differential Equations. Academic Press, San Diego (1999)

2. Kilbas, A.A., Srivastava, H.M., Trujillo, J.J.: Theory and Applications of Fractional Differential Equations. Elsevier, Amsterdam (2006)

3. Zhou, Y.: Basic Theory of Fractional Differential Equations. World Scientific, Singapore (2014)

4. Zhou, Y.: Fractional Evolution Equations and Inclusions: Analysis and Control. Academic Press, San Diego (2016)

5. Dong, $H_{\text {., Kim, } \mathrm{D} .:} L_{p}$-estimates for time fractional parabolic equations with coefficients measurable in time. Adv. Math. 345, 289-345 (2019)

6. Kim, I., Kim, K.H., Lim, S.: $L_{q}\left(L_{p}\right)$-theory for the time fractional evolution equations with variable coefficients. Adv. Math. $306,123-176(2017)$

7. Giga, Y., Namba, T.: Well-posedness of Hamilton-Jacobi equations with Caputo's time fractional derivative. Commun. Partial Differ. Equ. 42, 1088-1120 (2017)

8. Li, L., Liu, J.G., Wang, L.: Cauchy problems for Keller-Segel type time-space fractional diffusion equation. J. Differ. Equ. 265, 1044-1096 (2018)

9. Lin, C., Nakamura, G.: Unique continuation property for multi-terms time fractional diffusion equations. Math. Ann. 373, 929-952 (2019)

10. Li, K., Peng, J., Jia, J.: Cauchy problems for fractional differential equations with Riemann-Liouville fractional derivatives. J. Funct. Anal. 263, 476-510 (2012)

11. Shu, X.B., Wang, Q.Q.: The existence and uniqueness of mild solutions for fractional differential equations with nonlocal conditions of order $1<\alpha<2$. Comput. Math. Appl. 64, 2100-2110 (2012)

12. Li, Y.: Regularity of mild solutions for fractional abstract Cauchy problem with order $\alpha \in(1,2)$. Z. Angew. Math. Phys. 66, 3283-3298 (2015)

13. Kian, Y., Yamamoto, M.: On existence and uniqueness of solutions for semilinear fractional wave equations. Fract. Calc. Appl. Anal. 20, 117-138 (2017)

14. Li, Y., Sun, H., Feng, Z.: Fractional abstract Cauchy problem with order $\alpha \in(1,2)$. Dyn. Partial Differ. Equ. 13, 155-177 (2016)

15. Agarwal, R.P., Baleanu, D., Nieto, J.J., Torres, D.F.M., Zhou, Y.: A survey on fuzzy fractional differential and optimal control nonlocal evolution equations. J. Comput. Appl. Math. 339, 3-29 (2018)

16. Li, K., Peng, J., Gao, J.: Controllability of nonlocal fractional differential systems of order $\alpha \in(1,2]$ in Banach spaces. Rep. Math. Phys. 71, 33-43 (2013)

17. He, J.W., Peng, L.: Approximate controllability for a class of fractional stochastic wave equations. Comput. Math. Appl. 78, 1463-1476 (2019)

18. Wang, J., Zhou, Y.: A class of fractional evolution equations and optimal controls. Nonlinear Anal., Real World Appl. 12, 262-272 (2011)

19. Wang, J., Zhou, Y., Wei, W.: A class of fractional delay nonlinear integrodifferential controlled systems in Banach spaces. Commun. Nonlinear Sci. Numer. Simul. 16(10), 4049-4059 (2011)

20. Fan, Z., Mophou, G.: Existence and optimal control for fractional evolution equations. Nonlinear Stud. 20(2), 161-170 (2013)

21. Podlubny, I.: Fractional-order systems and $\left.P\right|^{\lambda} D^{\mu}$ controller. IEEE Trans. Autom. Control 44, 208-214 (1999)

22. Wang, J., Wei, W., Zhou, Y.: Fractional finite time delay evolution systems and optimal controls in infinite-dimensional spaces. J. Dyn. Control Syst. 17(4), 515-535 (2011)

23. Wang, J., Zhou, Y., Medved, M.: On the solvability and optimal controls of fractional integrodifferential evolution system with infinite delay. J. Optim. Theory Appl. 152(1), 31-50 (2012)

24. Chang, Y.K., Ponce, R.: Sobolev type time fractional differential equations and optimal controls with the order in $(1,2)$. Differ. Integral Equ. 32(9/10), 517-540 (2019)

25. He, J.W., Peng, L.: Approximate controllability for a class of fractional stochastic wave equations. Comput. Math. Appl. 78(5), 1463-1476 (2019)

26. He, J.W., Liang, Y., Ahmad, B., Zhou, Y.: Nonlocal fractional evolution inclusion of order $\alpha \in(1,2)$. Mathematics 7(2) , 209 (2019)

27. Zhou, Y., He, J.W.: New results on controllability of fractional evolution systems with order $\alpha \in(1,2)$. Evol. Equ. Control Theory (2020). https://doi.org/10.3934/eect.2020077 
28. Raja, M.M., Vijayakumar, V., Udhayakumar, R., Zhou, Y.: A new approach on the approximate controllability of fractional differential evolution equations of order $1<\gamma<2$ in Hilbert spaces. Chaos Solitons Fractals 141, 110310 (2020)

29. Raja, M.M., Vijayakumar, V., Udhayakumar, R.: Results on the existence and controllability of fractional integro-differential system of order $1<\gamma<2$ via measure of noncompactness. Chaos Solitons Fractals 139, 110299 (2020)

30. Raja, M.M. Vijayakumar, V., Udhayakumar, R.: A new approach on approximate controllability of fractional evolution inclusions of order $1<\gamma<2$ with infinite delay. Chaos Solitons Fractals 141, 110343 (2020)

31. Williams, W.K., Vijayakumar, V., Udhayakumar, R., Nisar, K.S.: A new study on existence and uniqueness of nonlocal fractional delay differential systems of order $1<\gamma<2$ in Banach spaces. Numer. Methods Partial Differ. Equ. (2020). https://doi.org/10.1002/num.22560

32. Gorbatenko, Y.V.: Existence and uniqueness of mild solutions of second order semilinear differential equations in Banach space. Methods Funct. Anal. Topol. 17(1), 1-9 (2011)

33. Xiang, X.L., Huawu, K.: Delay systems and optimal control. Acta Math. Appl. Sin. 16(1), 27-35 (2000)

34. Zeidler, E.: Nonlinear Functional Analysis and Its Application: I/B: Nonlinear Monotone Operators. Springer, Berlin (2013)

35. Goldstein, J.A.: Semigroups of Linear Operators and Applications. Oxford University Press, New York (1985)

36. Arendt, W., Batty, C.J.K., Hieber, M., Neubrander, F.: Vector-Valued Laplace Transforms and Cauchy Problems, 2nd edn. Birkhäuser, Basel (2011)

37. Travis, C.C., Webb, G.F.: Cosine families and abstract nonlinear second order differential equations. Acta Math. Hung. 32, 75-96 (1978)

38. Feckan, M., Wang, J., Zhou, Y.: Controllability of fractional functional evolution equations of Sobolev type via characteristic solution operators. J. Optim. Theory Appl. 156(1), 79-95 (2013)

\section{Submit your manuscript to a SpringerOpen ${ }^{\circ}$ journal and benefit from:}

- Convenient online submission

- Rigorous peer review

- Open access: articles freely available online

- High visibility within the field

- Retaining the copyright to your article

Submit your next manuscript at $\boldsymbol{\triangleright}$ springeropen.com 